\title{
HIGH-CAPACITY DATA HIDING IN HALFTONE IMAGES USING MINIMAL ERROR BIT SEARCHING
}

\author{
Soo-Chang Pei, Fellow, IEEE, and Jing-Ming Guo \\ Department of Electrical Engineering \\ National Taiwan University, Taipei, Taiwan 10617, R. O. C. \\ Tel: 886-2-23635251-321; Fax: 886-2-23671909

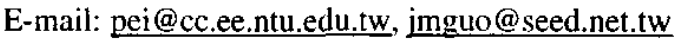

\begin{abstract}
In this paper, a high capacity data hiding is proposed for embedding a large amount of information into halftone images. The embedded watermark can be distributed into several errordiffused images by the proposed Minimal Error Bit Searching technique (MEBS). The method can be generalized to selfdecoding mode with dot diffusion or color halftone images. From the experiments, the embedded capacity from $33 \%$ up to $50 \%$ and good quality result are achieved. Furthermore, the proposed MEBS method is also extended for robust watermarking to against the degradation from printing-andscanning and several kinds of distortions.
\end{abstract}

\section{INTRODUCTION}

Digital halftoning [1] is to render multi-tone images using only two-tone elements. There are several kinds of halftoning methods, including ordered dithering, enror diffusion [2], dot diffusion [3]-[4]. Among these, error diffusion offers good visual quality and reasonable computational complexity, and the dot diffusion attempts to retain the good quality of error diffusion while offering substantial parallelism.

Data hiding in halftone images can be used for printing security documents such as ID card, currency as well as confidential documents, and they prevent from illegal duplication and forgery by further scanning these documents to digital forms.

Some of the published techniques include using a number of different dither cells to create a threshold pattern in the halftoning process [5], where one cell stands for one information bit; coordinating the $\mathrm{BCH}$ error correcting code with data hiding techniques [6], where the embedded capacity as high as $11 \%$ is achieved there; embedding hidden visual patterns into two or more halftone images, and the hidden visual patterns can be perceived directly when the halftone images are overlaid each other [7]. In [7], however, the visual decoded pattern is just an approximation of the original watermark. In this paper, we propose a new algorithm to precisely decode the watermark, and the embedded capacity is still kept high.

\section{THE QUALITY EVALUATION}

Let the size of the original image be $P \times Q$. Here we define 1 as a white pixel and 0 as a black pixel. The variable $x_{i, j}$ represents the gray level image pixel value at position $(i, j)$ and the variable $b_{i, j}$ means binary output. The quality evaluation in this paper is defined as below.

$$
\text { PSNR }=\frac{P \times Q}{\sum_{i=1}^{P} \sum_{j=1}^{Q}\left[x_{i, j}-\sum_{m, n \in R} \sum w_{m, n} b_{i+m, j+n}\right]^{2}}
$$

where $w_{m, n}$ is the human visual coefficient at position $(m, n)$, and $R$ is the support region of the human visual coefficients. In this paper we fixed $R$ at $15 \times 15$. The human visual filter $w$ can be obtained by psychophysical experiments [8]. The other way to derive $w$ uses a training set of both pairs of gray level images and good halftone results of them, such as using error diffusion or ordered dithering to produce the set. Here we use Least-Mean-Square (LMS) to derive $w$ as below.

$\bar{x}_{i, j}=\sum_{m, n \in R} \sum w_{m, n} b_{i+m, j+n}$,

$e_{i, j}^{2}=\left(x_{i, j}-\bar{x}_{i, j}\right)^{2}$,

$\frac{\partial e_{i, j}^{2}}{\partial w_{m, n}}=-2 e_{i, j} b_{i+m, j+n}$,

$\left\{\right.$ if $w_{m, n}>w_{m, n, v p t}$, slope $>0, w_{m, n}$ should be decreased

if $w_{m, n}<w_{m, n, p p t}$, slope $<0, w_{m, n}$ should be increased,

$w_{m, n}^{(k+1)}=w_{m, n}^{k}+\mu e_{i, j} b_{i+m, j+n}$, where $w_{i, j, o p t}$ is the optimum LMS coefficient; $\mu$ is the adjusting parameter used to control the convergent speed of the LMS optimum procedure, with the value set to $10^{-5}$ in this paper.

There are 8 training images used in our training process: Lena, Mandrill, Peppers, Milk, Airplane, Earth, Lake, and Tiffany images. The Floyd, Jarvis error diffusion kernels, as well as Bayer-5 ordered dithering are used to produce the corresponding halftone training results [1]-[2]. Due to the limitation of paragraph, we can not particularly list the final trained coefficients here. 


\section{DATA HIDING WITH MINIMAL ERROR BIT SEARCHING (MEBS)}

\subsection{Standard Minimal Error Bit Searching}

The encoding scheme proposed here is to distribute the desired watermark into several error diffusion (EDF) images. The decoding scheme can be applied only when these embedded EDF images are all collected already. The concept is similar to secret sharing scheme [9].

The proposed high capacity Minimal Error Bit Searching (MEBS) data hiding encoder is depicted in Fig. 1. Some variables are defined as follows. $x_{i, j}^{n}$ is the current input pixel value of $n^{t h}$ gray level image; $v_{i, j}^{n}$ is the modified gray output; $b_{i, j}^{n}$ is the temporary binary output; $b_{i, j}^{n}{ }^{\prime}$ is the final binary output after MEBS arrangement; $W_{i, j}$ is the original watermark data. $B_{i, j}$ is the temporary binary outputs vector, with the form of $\left\{b_{i, j}^{1}, b_{i, j}^{2}, \cdots, b_{i, j}^{n}\right\} ;$, is the modified binary outputs vector, with the form of $\left\{b_{i, j}^{\mathbf{i}}, b_{i, j}^{2}, \cdots, b_{i, j}^{\prime}{ }^{\prime}\right\}$, where $B_{i, j}^{\prime}$ differs from $B_{i, j}$ by only one bit value. The Jarvis error diffusion kernel $h_{m, n}$ [2] is used here. Here we use a modified binary output vector to embed one bit in the watermark.

Now we describe the Minimal Error Bit Searching (MEBS) algorithm. As illustrated in Table I, the vector dimension of 3 is taken as an example. We first generate the 3 bits Gray code, and address each codeword with a corresponding information bit 0 or 1. Note that the information bits 0 and 1 are arranged alternately, and the table is divided into two groups ( 0 -group and I-group). From this arrangement it is clear that if any one bit of a codeword in 0-group is changed, the new codeword will fall into the 1-group, and vice versa. If we want to embed information bit 0 into the host EDF images, then we check if the binary output vector $B_{i, j}$ is mapped to 0-group, if yes, then no bit in this vector should be varied, if no, then we just need to modify one bit in this vector. The proposed minimal error bit searching is used for judging which bit in the vector is the most suitable candidate. Here we define other variables $e_{i, j}^{n}$ and $b_{i, j}^{n} "$ as follows.

$e_{i, j}^{n}=v_{i, j}^{n}-b_{i, j}^{n}$,

where $b_{i, j}^{n}=\left\{\begin{array}{lll}1 & \text { if } & v_{i, j}^{n}<0.5 \\ 0 & \text { if } \quad v_{i, j}^{n} \geq 0.5,\end{array} \quad n=1,2,3, \cdots\right.$

The modified binary outputs vector is formed as below.

$B_{i, j}^{\prime}=\left\{b_{i, j}^{1}, b_{i, j}^{2}, \cdots, b_{i, j}^{k}, \cdots, b_{i, j}^{n}\right\}$,

where $e_{i, j}^{k^{\prime}}=\min \operatorname{imum}\left\{e_{i, j}^{j^{\prime}}, e_{i, j}^{2^{\prime}}, \cdots, e_{i, j}^{n}\right\}$, and $k \in\{1, \cdots, n\}$. In the decoder, we just need to collect the corresponding bits in these embedded images and form into the decoded vector sets, then use the Table Look Up method (LUT) for decoding. For example, from the Table $I$, the decoded vector of $\{1,0,1\}$ represents that an information bit "l" had been embedded.

\subsection{Modified Minimal Error Bit Searching}

From the expcriments, we find that when the capacity gets as bigh as $50 \%$, a large amount of output bits $b_{i, j}$, with higher error difference $e_{i, j}$, is forced to be diffused. This sort of phenomenon will degrade the embedded image quality. So we propose a modified MEBS to overcome this problem. Equation (7) can be modified as:

$e_{i, j}^{n}=\left\{\begin{array}{lr}E R R_{t h}, & \text { if }\left|E R R_{t h}\right|<\mid v_{i, j}-b_{i, j}^{n} " \\ v_{i, j}-b_{i, j}^{n}, & \text { otherwise, }\end{array}\right.$

where $E R R_{t h}$ is the pre-defined error threshold with the value set to 100 in this paper. With this strategy, the output bits with unreasonable high error differences are avoided.

\section{SELF-DECODING MODE AND WATERMARKING EXTENSION}

\subsection{Dot diffusion self-decoding mode}

In [4], Mese and Vaidyanathan improved the quality of dotdiffused images by optimizing the class matrix. The modified class matrix is also adopted in this paper. Due to the limitation of paragraph, we do not describe the algorithm of dot diffusion here.

The dot diffusion inherently has the benefit of parallelism. We can arbitrarily divide the original gray level image to 2 or more portions. Each portion is taken as one host image then cooperate the dot diffusion with the proposed MEBS to embed watermark into one image.

\subsection{Color halftone image self-decoding mode}

In addition to the dot diffusion, because the color image inherently has several color spaces, e. g., R-G-B or C-M-Y-K, it can also be applied for the self-decoding mode. Each color space stands for one host image in MEBS encoded process. However, all the color spaces have very high correlations with each other. So if we directly embed the watermark by MEBS technique, some high intensity regions in color embedded halftone image will be noisy. In this paper, we simply reverse the even color spaces to solve this problem. The even color spaces in C-M-Y-K is $M$ and $K$, as well as $G$ in R-G-B. The new color spaces of the color image are as below

$$
\left(x_{i, j}^{n}\right)_{n e w}=x_{i, Q-j}^{n} \text {, if }(\mathrm{n} \bmod 2)=0
$$

After encoding, the even color spaces are reversed again to form the normal embedded color halftone image.

\subsection{Robust watermarking extension}

The original embedded halftone watermarked images are usually degraded by several kinds of attacks or distortions. The proposed MEBS technique can be extended to robust watermarking to against such degradation. Here we divide each original gray 
level images of size $P \times Q$ into several cells of size $M_{0} \times N_{0}$. In each cell we embed the same information bit, and these host images can embed a bi-level watermark of size $\left(\frac{P}{M_{0}}\right) \times\left(\frac{Q}{N_{0}}\right)$.

Then we can use the majority voting to recover the damaged portion caused by all kinds of distortions.

\section{EXPERIMENTAL RESULTS}

Figure 2 represents one original bi-level watermark and three original error-diffused halftone images (Lena, Mandrill, and color Lena), respectively, which are of size $512 \times 512$, and printed at $300 \mathrm{dpi}$. The PSNR of three original error-diffused halftone images are $30.84,27.15$, and $30.92 \mathrm{~dB}$, respectively. Figures 3(a)-(b) are obtained by embedding the watermark into Fig. 2(b)-(c) with the standard MEBS, and the PSNR are 25.6 and 25.45 , respectively. Although the capacity here is $50 \%$, degradation in the quality is obvious found. For this, we apply the proposed modified MEBS as described in Section 3.2 to solve the problem. The modified embedded images are shown in Fig. 3(c)-(d), and the PSNR are 26.2 and 25.97, respectively. We also tested for increasing the number of host images to three. The corresponding PSNR for embedded images Lena, Mandrill, and Peppers are 28.9, 27.6, and $28.95 \mathrm{~dB}$, respectively. Since the watermark is shared into three images at the same size as the watermark, the capacity is $33.33 \%$.

Figures $4(\mathrm{a})$-(c) represent one original $480 \times 480$ dotdiffused Lena image (PSNR $=30.8 \mathrm{~dB}$ ) and two watermarks of sizes $240 \times 480$ and $160 \times 480$, respectively. Figure $4(d)$ $(P S N R=25.39 \mathrm{~dB}$ ) is obtained by embedding Fig. 4 (b) into Fig. 4(a) with modified MEBS technique, and the corresponding capacity is $50 \%$. In the same way, the original $480 \times 480$ Lena image can be divided into three portions. The Fig. 4(c) watermark with one-third size can be embedded into full size Lena image to form the result shown in Fig. 4(e) $(P S N R=28.84 \mathrm{~dB})$, and the corresponding capacity is $33.33 \%$.

Figure 5(a) (PSNR=29.75) is obtained by embedding Fig. 2(a) watermark into four color spaces of color Lena image with even color spaces reversing as described Section 4-2.

Following we discuss the performance of robust watermarking extension of the proposed MEBS technique. The watermark of size $64 \times 64$ is used. The divided cell size $M_{0} \times N_{0}$ described in Section 4-3 is $8 \times 8$, and the color spaces used here are C, M, Y, and K. Fig. $5(\mathrm{~b})$ is the embedded images attacked with tampering, and the correct decoded rate is 98.54 . We also tested the cropping $1 / 4$ and $2 / 5$. The decoded rates are 99.83 and 96.29 , respectively.

It is very difficult to perfectly extract the original watermarks from a printed-and-scanned halftoned image. We geometrically transform the printed-and-scanned embedded image into size $512 \times 512$ before the decoding process, then divided into $512 \times 512$ square blocks, and the average of the pixels within a block is thresholded to recover the original halftone image pixel $(0$ or 1$)$. In Table II we show the average correct decoded rates for 8 printed-and-scanned tested images, which including Lena, Mandrill, Peppers, Milk, Tiffany, Earth, Lake, and Airplane images.
From the experimental results discussed above, we can prove that the proposed waternarking is robust to survive under various attacks and distortions, such as cropping, tampering, and printing-and-scanning etc.

\section{REFERENCES}

[1] R. Ulichney, Digital Halftoning. Cambridge, MA. MIT Press, 1987.

[2] J. F. Jarvis, C. N. Judice, and W. H. Ninke, “A survey of techniques for the display of continuous-tone pictures on bilevel displays," Comp. Graph. Image Proc., vol. 5, pp. 1340,1976

[3] D. E. Knuth, "Digital halftones by dot diffusion," $A C M$ Trans. Graph., vol. 6, no. 4, Oct. 1987.

[4] M. Mese and P. P. Vaidyanathan, "Optimized halftoning using dot diffusion and methods for inverse halftoning," IEEE. Trans. Image Proc., vol. 9, no. 4, pp. 691-709, April 2000.

[5] H. Z. HeI-Or, "Watermarking and copyright labeling of printed images," in Journal of Electronic Imaging, vol. 10(3), pp. 794-803, July 2001.

[6] M. S. Fu and O. C. Au, "Data Hiding watermarking for halftone images," IEEE Trans. Image Processing, vol. 11, pp. 477-484, April 2002.

[7] M. S. Fu and O. C. Au, "Data hiding in halftone images by stochastic error diffusion," in Proceedings of IEEE International Conference on Acoustics, Speech and Signal Processing, May 2000.

[8] J. Mannos and D. Sakrison, "The effects of a visual fidelity criterion on the encoding of images," IEEE Trans. Inform. Theory, vol. 20, pp. 526-536, 1974.

[9] A. Shamir, "How to share a secret." Communications of the ACM 22 (1979), 612-613

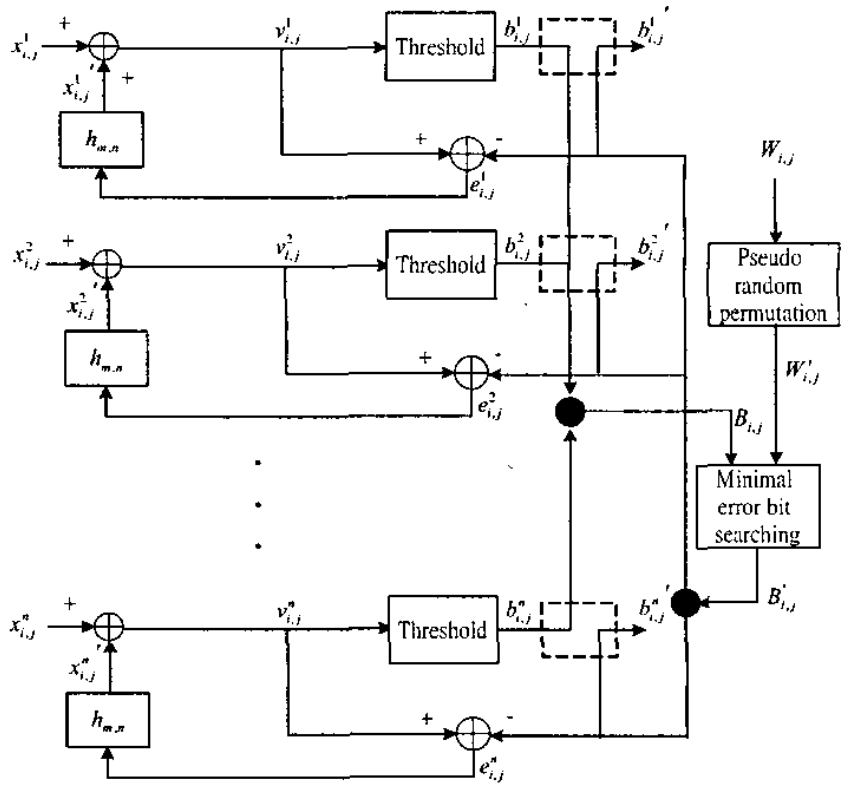

Fig. 1. Proposed high capacity Minimal Error Bit Searching (MEBS) data hiding encoder. 


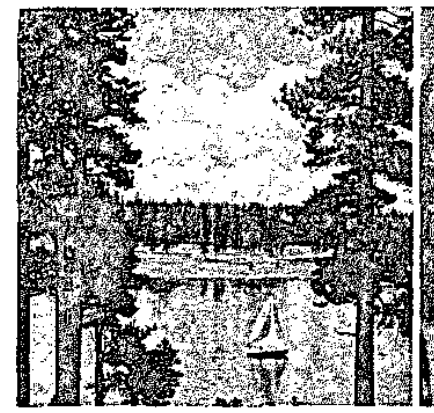

(a)

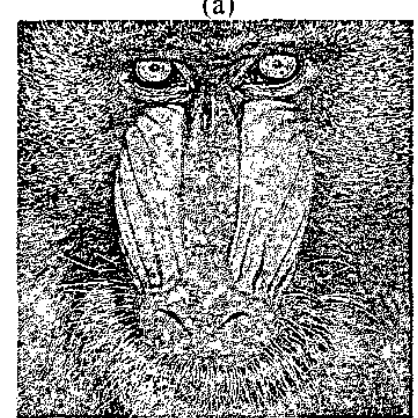

(c) $P S N R=27.15 \mathrm{~dB}$

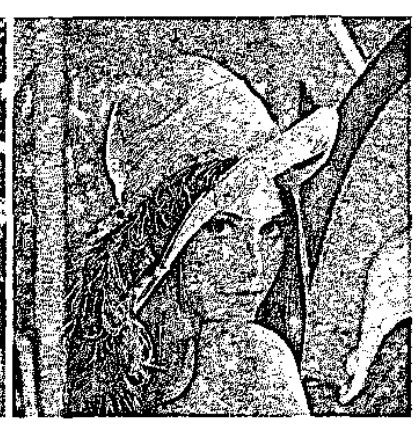

(b) $\mathrm{PSNR}=30.84 \mathrm{~dB}$

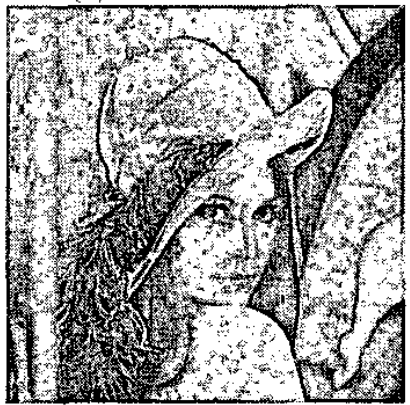

(d)PSNR $=30.92 \mathrm{~dB}$

Fig. 2. (a) Original bi-level watemark $(512 \times 512)$. (b)-(d) Original error-diffused halftone images (b) Lena (c) Mandrill (d) color Lena.

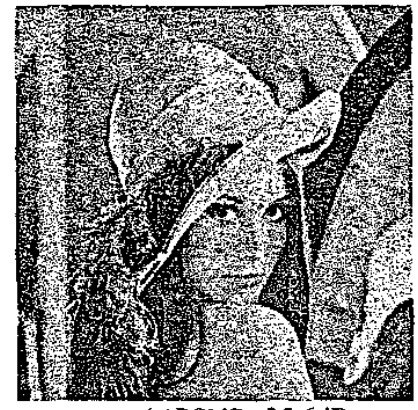

(a)PSNR $=25.6 \mathrm{~dB}$

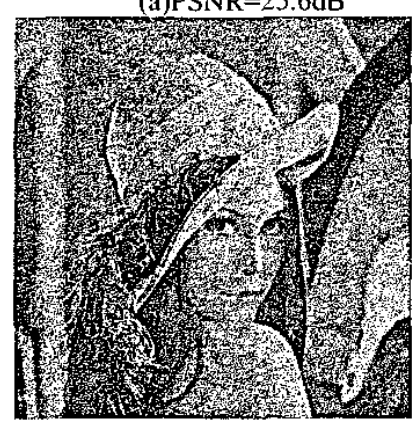

(a) $P S N R=26.2 \mathrm{~dB}$

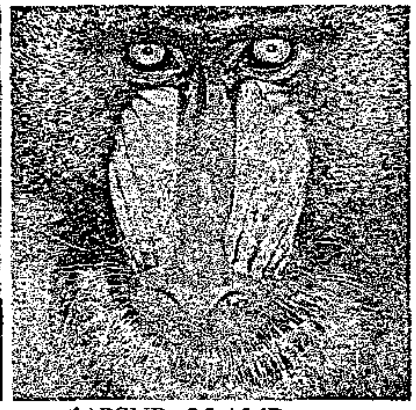

(b) $P S N R=25.45 \mathrm{~dB}$

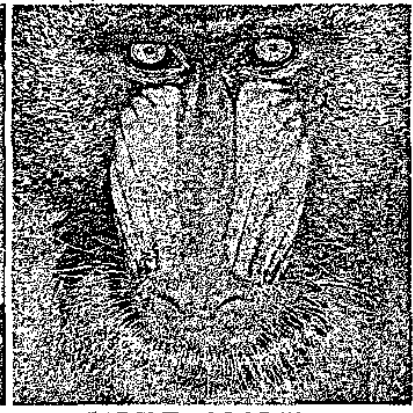

(b) $P S N R=25.97 \mathrm{~dB}$

Fig. 3. (a)-(b) Obtained by distributing the Fig.2(a) watermark into Fig. 2(b)-(c) with standard MEBS. (c)-(d) Obtained by distributing the Fig.2(a) watermark into Fig. 2(b)-(c) with modified MEBS.

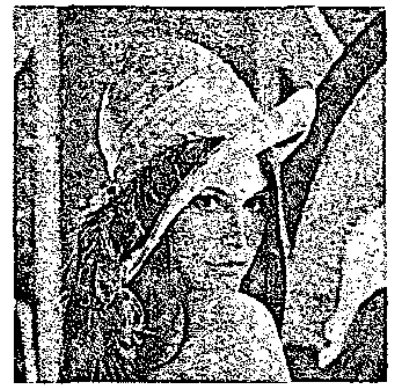

(i) $\mathrm{PSNR}=30.6 \mathrm{~dB}$

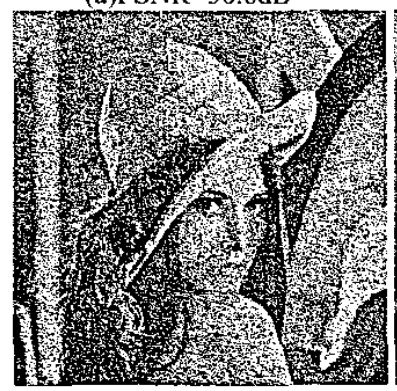

(d)PSNR $=25.39 \mathrm{~dB}$

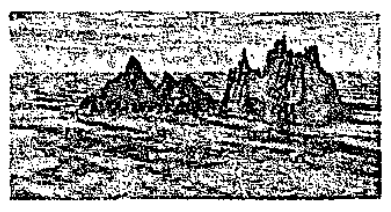

(b)

(c)

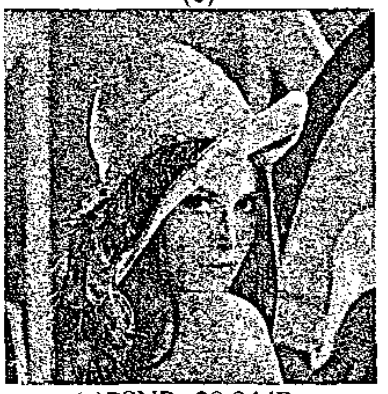

(e)PSNR $=28.84 \mathrm{~dB}$

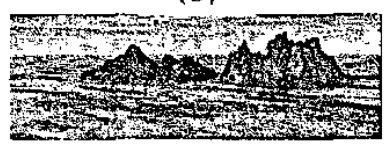

Fig. 4. (a) $480 \times 480$ dot-diffused image. (b)-(c) Watermark with size $240 \times 480$ and $160 \times 480$. (d) Embed (b) to (a) with modified MEBS. (e) Embed (c) to (a) with MEBS.

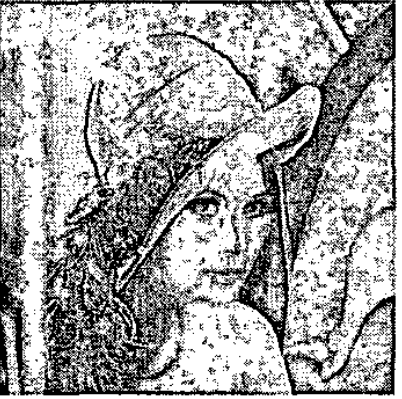

(a) $\mathrm{PSNR}=29.75 \mathrm{~dB}$

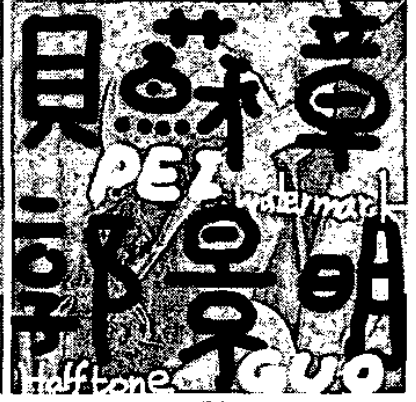

(b)
Fig. 5. (a) Embedded color Lena image with even colors space reversing. (b) Attack the embedded image with tampering.

TABLE I. THREE BITS GRAY CODE AND THE
CORRESPONDING INFORMATION BITS
\begin{tabular}{|c|c|c|c|c|c|c|c|c|c|}
\hline Information bit & 1 & 0 & 1 & 0 & 1 & 0 & 1 & 0 \\
\hline \multirow{3}{*}{ Gray code } & 0 & 1 & 1 & 0 & 0 & 1 & 1 & 0 \\
\hline & 0 & 0 & 1 & 1 & 1 & 1 & 0 & 0 \\
\hline & 0 & 0 & 0 & 0 & 1 & 1 & 1 & 1 \\
\hline
\end{tabular}

TABLE. II. AVERAGE CORRECT DECODING RATES OF 8 TESTED IMAGES AFTER PRINT-AND-SCAN.

\begin{tabular}{|c|c|c|}
\hline \multicolumn{2}{|c|}{ Embedded image form } & Average decoding rates \\
\hline \multicolumn{2}{|c|}{ Bitmap } & $100 \%$ \\
\hline \multirow{3}{*}{ Scanned } & $750 \mathrm{dpi}$ & $86.59 \%$ \\
\cline { 2 - 3 } & $450 \mathrm{dpi}$ & $83.52 \%$ \\
\cline { 2 - 3 } & $150 \mathrm{dpi}$ & $76.13 \%$ \\
\hline
\end{tabular}

